\title{
High economic burden of immunotherapy underlines the need of predictive biomarkers for the individual therapy algorithm in metastatic bladder cancer
}

\author{
Renate Pichler ${ }^{1}$, Wolfgang Loidl' ${ }^{2}$ Martin Pichler ${ }^{3,4}$ \\ ${ }^{1}$ Department of Urology, Medical University of Innsbruck, Innsbruck, Austria; ${ }^{2}$ Department of Urology, St Vincent's Hospital of Linz, Linz, Austria; \\ ${ }^{3}$ Division of Clinical Oncology, Internal Medicine, Medical University of Graz, Graz, Austria; ${ }^{4}$ Division of Cancer Medicine, MD Anderson Cancer \\ Center, Houston, TX, USA \\ Correspondence to: Assoc. Prof. Renate Pichler, MD, PhD. Department of Urology, Urologic Oncology, Medical University of Innsbruck, Anichstreet \\ 35, A-6020 Innsbruck, Austria. Email: Renate.Pichler@i-med.ac.at. \\ Provenance: This is an invited Editorial commissioned by Section Editor Xiao Li (Department of Urology, Jiangsu Cancer Hospital \& Jiangsu \\ Institute of Cancer Research \& Nanjing Medical University Affiliated Cancer Hospital, Nanjing, China). \\ Comment on: Sarfaty M, Hall PS, Chan KKW, et al. Cost-effectiveness of Pembrolizumab in Second-line Advanced Bladder Cancer. Eur Urol \\ 2018;74:57-62.
}

Submitted Aug 09, 2018. Accepted for publication Aug 13, 2018.

doi: $10.21037 /$ tau.2018.08.15

View this article at: http://dx.doi.org/10.21037/tau.2018.08.15

Bladder cancer is a highly immunogenic and heterogeneous tumor (1). Its responsiveness to immunotherapy is well known since the successful introduction of intravesical BCG in 1976 (2). This procedure still is the standard adjuvant treatment for high-risk non-muscle-invasive bladder cancer (3).

In 2016, five checkpoint inhibitors were approved by the US Food and Drug Administration (FDA) as secondline treatment for metastatic bladder cancer. Of these, pembrolizumab was the only drug that yielded an overall survival (OS) benefit according to the KEYNOTE-045 study (4). This novel therapeutic approach has revolutionized the care of patients with bladder cancer, transforming a highly lethal and aggressive disease into one that can be managed by persistent tumor control in some patients, a better tolerance and a better safety profile than chemotherapy $(4,5)$.

The steadily growing economic burden of immunotherapy has become a matter of concern for public and private funding agencies as well as policy makers. Validated analyses of the cost-effectiveness of immunotherapy have become a point of focus. Sarfaty et al. (6) evaluated, for the first time, the cost-effectiveness of pembrolizumab versus second-line chemotherapy (docetaxel, paclitaxel, and vinflunine only for the UK model) from the perspective of payers; four countries (United States, United Kingdom, Canada and Australia) were included in the analysis. Interestingly, prices varied significantly around the world because of diverse regulations and negotiations with drug companies; the highest costs were noted in the US (122,557 \$/quality adjusted life-years). In other words, a single cycle of treatment with pembrolizumab is 15 to 50 times more expensive than chemotherapy. In view of the highest willingness-to-pay threshold (100,000 to 150,000 \$/quality adjusted life-years) in the US, pembrolizumab appears to be cost-effective only in the US and not in other countries. As Sarfaty and coworkers (6) have mentioned, there may be several reasons for underestimating the survival benefit of pembrolizumab: (I) no crossover analysis was performed, although at least $12.9 \%$ of patients in the chemotherapy arm received immunotherapy as third-line treatment; (II) real-world patients may not be comparable with trial participants. Due to its lower toxicity rates, pembrolizumab will be used in the clinical setting more often in multimorbid patients with a poor performance status; (III) continuation of immunotherapy despite radiographic progression at first evaluation, as a measure to counteract "pseudo-progression", is a common approach in many centers; the reason is that approved third-line therapies 
after immunotherapy do not exist at the present time.

The study by Sarfaty et al. (6) underlines the urgent need for further analyses of cost-effectiveness with a comparison of all five FDA-approved (pembrolizumab, nivolumab, atezolizumab, avelumab, durvalumab) and all three EMAapproved (pembrolizumab, nivolumab, atezolizumab) second-line checkpoint inhibitors. This will be necessary before one can draw any final conclusions about the costeffectiveness of pembrolizumab compared to avelumab, atezolizumab, nivolumab, or durvalumab.

The recent introduction of checkpoint inhibitors in the management of advanced bladder cancer has expanded the range of treatment options considerably. Selection of the ideal agent for each patient and sequencing the agent in the current treatment paradigm and algorithm continue to pose a major challenge in the clinical practice. Predictive biomarkers are urgently needed not only to select those patients who are most likely to benefit from immunotherapy, but to reduce the cost of subsequent treatment. Only about a third of patients will have a sustained response to checkpoint inhibitors, independent of PD-L1 expression $(4,5,7)$. Responses have also been noted in PD-L1-negative bladder cancer patients, thus confirming a poor negative predictive value of PD-L1 $(4,5,7)$. Preliminary results from the ongoing KEYNOTE-361 (NCT02853305) and IMvigor130 trial (NCT02807636) showed reduced survival in the checkpoint inhibitor monotherapy arm compared with standard platinum-based chemotherapy in the first-line setting, and thus restricting immunotherapy to cisplatinunfit patients with high PD-L1 expression (8). In non-small cell lung cancer, PD-L1 expression as a predictive marker in the second-line treatment of NSCLC increased the costeffectiveness of immunotherapy, but with the disadvantage to reduce the number of potential life-years saved (9).

Tumor mutational burden (10), APOBEC mRNA expression profiling $(11,12)$, next-generation sequencing of cell-free circulating tumor DNA (13) and the Cancer Genome Atlas (TGCA) molecular subtyping of invasive bladder cancer (14) may brought about considerable clarity in the field of biomarker research.

Of the TGCA molecular subtypes, the luminal papillary I subtype which is marked by fibroblast growth factor receptor 3 (FGFR) alterations or mutations $(12,14,15)$ is a good example of an immunologically "cold" tumor (16). These tumors have shown no definitive response to checkpoint inhibitors and are thus no ideal candidates for checkpoint inhibitors (16); rather, they may be treated with other "targeted therapies" such as FGFR inhibitors introducing a novel targeted substance class in the treatment of metastatic bladder cancer (17). This fact has been corroborated by Joerger et al. (18) and Siefker-Radtke et al. (19), who presented their data at the ASCO annual meeting this year. The scientists evaluated the efficacy of the FGFR inhibitors rogaratinib and erdafitinib in heavily pretreated bladder cancer patients with FGFR mutations. Erdafitinib was associated with an objective response rate (ORR) of $40.4 \%$ and a median OS of 13.8 months, which is superior to that of second-line pembrolizumab (10.1 months) (4). Moreover, other targeted drugs such as enfortumab showed similar results in a phase I trial, with an overall ORR of $41 \%$ and a median OS of 13.6 months; it was also effective in patients who had undergone prior immuno-oncology therapy (ORR 40\%) (20). Based on these data, erdafitinib and enfortumab are being investigated further for secondand third-line treatment of metastatic bladder cancer, with the aim of finding the right treatment algorithm for the individual patient. In the phase III THOR trial, erdafitinib is compared with pembrolizumab and chemotherapy in patients with FGFR alterations (NCT03390504). The phase III EV-301 trial focuses on enfortumab versus chemotherapy in patients who have received prior platinum-based chemotherapy and experienced disease progression during treatment with checkpoint inhibitors (NCT03474107).

Finally, potent genetic and epigenetic biomarkers constitute a backbone of a promising approach to identify those patients who will most likely benefit from chemotherapy, checkpoint inhibitors or targeted therapies. The novel therapeutic approach with pan-FGFR inhibitors in metastatic bladder cancer patients with FGFR alterations or mutations represents the first step forwards for a more efficient personalized precision medicine.

\section{Acknowledgements}

None.

\section{Footnote}

Conflicts of Interest: The authors have no conflicts of interest to declare.

\section{References}

1. Wang L, Wheeler DA. Genomic sequencing for cancer diagnosis and therapy. Annu Rev Med 2014;65:33-48. 
2. Morales A, Eidinger D, Bruce AW. Intracavitary Bacillus Calmette-Guerin in the treatment of superficial bladder tumors. J Urol 1976;116:180-3.

3. Babjuk M, Böhle A, Burger M, et al. EAU Guidelines on Non-Muscle-invasive Urothelial Carcinoma of the Bladder: Update 2016. Eur Urol 2017;71:447-61.

4. Bellmunt J, de Wit R, Vaughn DJ, et al. Pembrolizumab as Second-Line Therapy for Advanced Urothelial Carcinoma. N Engl J Med 2017;376:1015-26.

5. Powles T, Durán I, van der Heijden MS, et al. Atezolizumab versus chemotherapy in patients with platinum-treated locally advanced or metastatic urothelial carcinoma (IMvigor211): a multicentre, open-label, phase 3 randomised controlled trial. Lancet 2018;391:748-57.

6. Sarfaty M, Hall PS, Chan KKW, et al. Cost-effectiveness of Pembrolizumab in Second-line Advanced Bladder Cancer. Eur Urol 2018;74:57-62.

7. Balar AV, Galsky MD, Rosenberg JE, et al. Atezolizumab as first-line treatment in cisplatin-ineligible patients with locally advanced and metastatic urothelial carcinoma: a single-arm, multicentre, phase 2 trial. Lancet 2017;389:67-76.

8. Gourd E. EMA restricts use of anti-PD-1 drugs for bladder cancer. Lancet Oncol 2018;19:e341.

9. Aguiar PN Jr, Perry LA, Penny-Dimri J, et al. The effect of PD-L1 testing on the cost-effectiveness and economic impact of immune checkpoint inhibitors for the secondline treatment of NSCLC. Ann Oncol 2017;28:2256-63.

10. Liu D, Abbosh P, Keliher D, et al. Mutational patterns in chemotherapy resistant muscle-invasive bladder cancer. Nat Commun 2017;8:2193.

11. Mullane SA, Werner L, Rosenberg J, et al. Correlation of Apobec Mrna Expression with overall Survival and pd-11 Expression in Urothelial Carcinoma. Sci Rep 2016;6:27702.

12. Robertson AG, Kim J, Al-Ahmadie H, et al. Comprehensive Molecular Characterization of Muscle-

Cite this article as: Pichler R, Loidl W, Pichler M. High economic burden of immunotherapy underlines the need of predictive biomarkers for the individual therapy algorithm in metastatic bladder cancer. Transl Androl Urol 2018;7(Suppl 6):S738-S740. doi: 10.21037/tau.2018.08.15
Invasive Bladder Cancer. Cell 2017;171:540-56.e25. Erratum in: Cell 2018;174:1033.

13. Barata PC, Koshkin VS, Funchain P, et al. Nextgeneration sequencing (NGS) of cell-free circulating tumor DNA and tumor tissue in patients with advanced urothelial cancer: a pilot assessment of concordance. Ann Oncol 2017;28:2458-63.

14. Cancer Genome Atlas Research Network. Comprehensive molecular characterization of urothelial bladder carcinoma. Nature 2014;507:315-22.

15. Kamat AM, Hahn NM, Efstathiou JA, et al. Bladder cancer. Lancet 2016;388:2796-810. Erratum in: Lancet 2016;388:2742.

16. Sharma P, Retz M, Siefker-Radtke A, et al. Nivolumab in metastatic urothelial carcinoma after platinum therapy (CheckMate 275): a multicentre, single-arm, phase 2 trial. Lancet Oncol 2017;18:312-22.

17. Lamont FR, Tomlinson DC, Cooper PA, et al. Small molecule FGF receptor inhibitors block FGFR-dependent urothelial carcinoma growth in vitro and in vivo. $\mathrm{Br} \mathrm{J}$ Cancer 2011;104:75-82.

18. Joerger M, Cassier PA, Penel N, et al. Rogaratinib in patients with advanced urothelial carcinomas prescreened for tumor FGFR mRNA expression and effects of mutations in the FGFR signaling pathway. J Clin Oncol 2018;36:abstr 4513.

19. Siefker-Radtke AO, Necchi A, Park SH, et al. First results from the primary analysis population of the phase 2 study of erdafitinib (ERDA; JNJ-42756493) in patients (pts) with metastatic or unresectable urothelial carcinoma (mUC) and FGFR alterations (FGFRalt). J Clin Oncol 2018;36:abstr 4503.

20. Rosenberg JE, Sridhar SS, Zhang J, et al. Updated results from the enfortumab vedotin phase 1 (EV-101) study in patients with metastatic urothelial cancer (mUC). J Clin Oncol 2018;36:abstr 4504. 